\title{
A Computer Scientist Looks at Game Theory
}

\author{
Joseph Y. Halpern* \\ Cornell University \\ Computer Science Department \\ Ithaca, NY 14853 \\ halpern@cs.cornell.edu \\ http://www.cs.cornell.edu/home/halpern
}

October 23, 2018

\begin{abstract}
I consider issues in distributed computation that should be of relevance to game theory. In particular, I focus on (a) representing knowledge and uncertainty, (b) dealing with failures, and (c) specification of mechanisms. Journal of Economic Literature Classification Numbers: D80, D83.
\end{abstract}

\section{Introduction}

There are many areas of overlap between computer science and game theory. The influence of computer science has been felt perhaps most strongly through complexity theory. Complexity theory has been viewed as a tool to help capture bounded rationality, going back to the work of Neyman [1985] and Rubinstein [1986]. In addition, it is well understood that complexity-theoretic notions like NP-completeness help categorize the intrinsic difficulty of a problem. Thus, for example, a result showing that, even in simple settings, the problem of optimizing social welfare is NP-hard Kfir-Dahav, Monderer, and Tennenholtz 2000 shows that the standard procedure of applying Clarke's mechanism, despite its many benefits, is not going to work in large systems.

Perhaps less obvious is the interplay between game theory and work in distributed computing. At the surface, both areas are interested in much the same problems: dealing with systems where there are many agents, facing uncertainty, and having possibly

${ }^{*}$ This paper is based on an invited talk I gave at Games 2000 in Bilbao, Spain. Supported in part by NSF under grants IRI-96-25901 and IIS-0090145 and by ONR under grants N00014-00-1-03-41, N0001401-10-511, and N00014-01-1-0795. 
different goals. In practice, however, there has been significant difference in emphasis in the two areas. In distributed computing, the focus has been on problems such as fault tolerance, scalability, and proving correctness of algorithms; in game theory, the focus has been on strategic concerns (that is, playing so as to optimize returns, in light of the preferences of other agents). In this paper, I hope to make the case that each area has much to learn from the other. I focus on three particular topics:

- the representation of games (and, in particular, the knowledge and uncertainty of players in a game),

- strategic concerns vs. fault tolerance, and

- specification of mechanisms.

The following sections deal with each of these topics in turn.

\section{Representing Games as Systems}

In order to analyze a game, we must first represent it. The two most common representations in the literature are the normal-form representation and the extensive-form representation. As is well known, the extensive-form representation brings out the temporal aspects of the game better, as well as explicitly representing (at least some aspects) of the players' knowledge. Consider the game that is represented in Figure 1 in both normal form and extensive form. The extensive-form representation brings out clearly that the game takes place over time, with the first player's second move, for example, occurring after the second player's first move. Moreover, when the first player makes that second move, he does not know what the second player's move is. However, as is also well known, the information sets used in the extensive-form representation do not capture all aspects of a player's information. For example, they cannot be used to capture beliefs one player has about what strategy the other player is using, or notions like rationality and common knowledge of rationality. The state-space representation does better in this regard.

\subsection{The state-space representation}

The state-space representation, first used in the economics literature by Aumann [1976, is actually a variant of the standard possible-worlds model for knowledge in the philosophical literature that goes back to Hintikka [1962]; see [Fagin, Halpern, Moses, and Vardi 1995, Section 2.5] for discussion. In this representation, each state in a state space $\Omega$ is a complete description of the world, which includes what happened in the past and what will happen in the future, the agents' beliefs and knowledge, and so on. 


\begin{tabular}{||l|ll||}
\hline & $\mathrm{A}$ & $\mathrm{D}$ \\
\hline aa & $(3,3)$ & $(4,2)$ \\
ad & $(3,2)$ & $(4,2)$ \\
da & $(1,2)$ & $(1,3)$ \\
dd & $(1,2)$ & $(1,3)$ \\
\hline
\end{tabular}

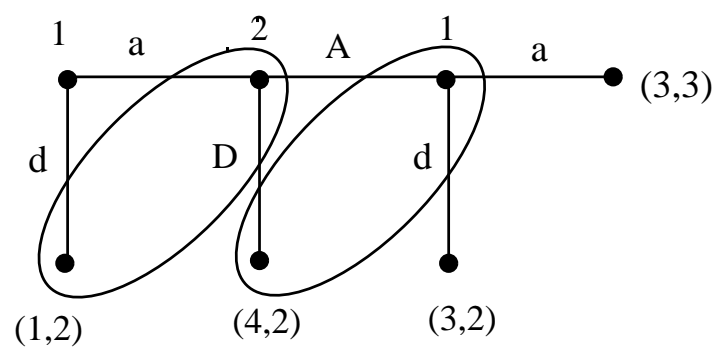

Figure 1: Representing a game in normal form and in extensive form.

One representation of the game in Figure 11 using a state space is given in Figure 2 . Let $\Omega=\left\{w_{1}, \ldots, w_{5}\right\}$. With each state $w \in \Omega$ is associated the strategy profile $\mathbf{s}(w)$ played at $w$. In this example,

- $\mathbf{s}\left(w_{1}\right)=\mathbf{s}\left(w_{5}\right)=(a a, A)$

- $\mathbf{s}\left(w_{2}\right)=(a a, D)$

- $\mathbf{s}\left(w_{3}\right)=(a d, A)$

- $\mathbf{s}\left(w_{4}\right)=(a d, D)$

In addition, there are two partitions associated with this state space, one for player 1 (denoted by ellipses in Figure 2) and one for player 2 (denoted by rectangles). The fact that $w_{3}$ and $w_{4}$ are both in the same cell of player 1's partition means that player 1 can't tell, in state $w_{3}$, if the actual state is $w_{3}$ or $w_{4}$. Note that in every cell for player 1 , player 1 is following the same strategy; similarly for player 2 . This is meant to capture the intuition that the players know their strategy. Further note that not all strategy profiles are associated with a state (for example, $(d d, A)$ is not associated with any state) and some profiles (such as $(a a, A)$ in this case) can be associated with more than one state. There is more to a state than the strategy profile used there. For example, in $w_{5}$, player 2 knows that the strategy profile is $(a a, A)$, while in $w_{1}$, player 2 considers it possible that $(a d, A)$ is played.

The state-space representation suffers from some of the same problems as the normalform representation. While it does a reasonably good job of capturing an agent's knowledge, it does not do such a good job of describing the play of the game - who moves 


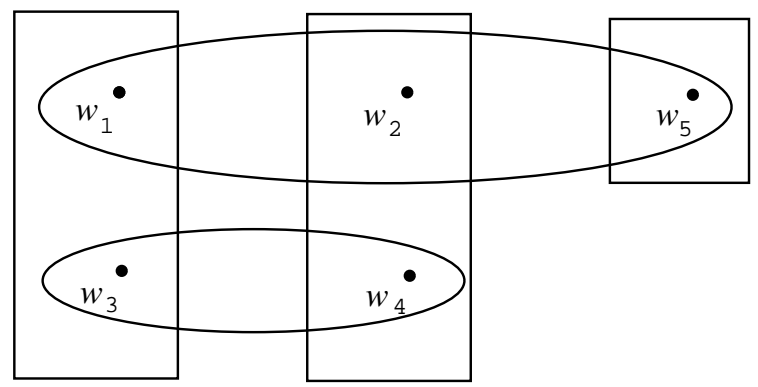

Figure 2: Representing the game of Figure 11 using a state space.

when, and what the possible moves are. Moreover, because time is not explicit in this representation, it becomes difficult to model statements such as "I know now that after I move my opponent will not know ...". More seriously, I would claim, neither the state-space representation nor the extensive-form representation makes it clear where the knowledge is coming from. Exactly what does it mean put two nodes or two states in the same information set?

This issue becomes particularly relevant when considering games with imperfect recall. Considering the single-agent game described in Figure 3, introduced by Piccione and Rubinstein [1997]. It is a game of imperfect recall since at the information set $\left\{x_{3}, x_{4}\right\}$, the agent has forgotten nature's initial move (i.e., whether it was earlier at $x_{1}$ or $x_{2}$ ).

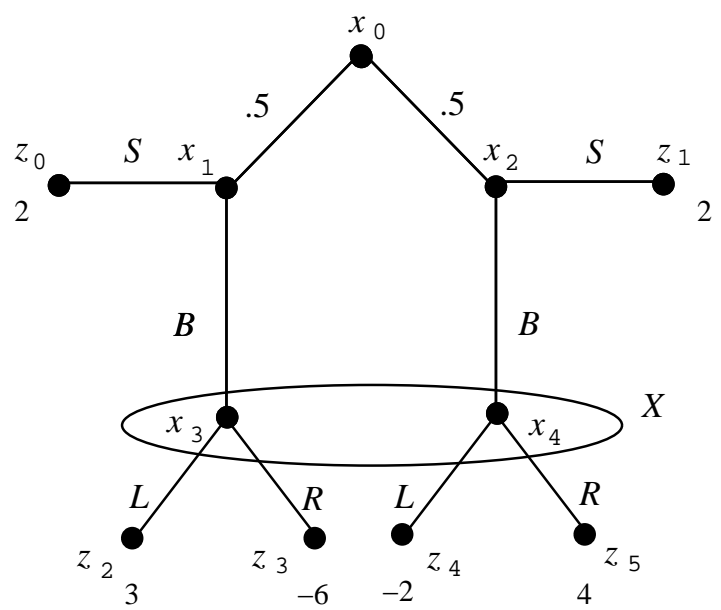

Figure 3: A game with imperfect recall.

It is not hard to show that the strategy that maximizes expected utility in this game chooses action $S$ at node $x_{1}$, action $B$ at node $x_{2}$, and action $R$ at the information set $X$ consisting of $x_{3}$ and $x_{4}$. Call this strategy $f$. Let $f^{\prime}$ be the strategy of choosing action $B$ at $x_{1}$, action $S$ at $x_{2}$, and $L$ at $X$. Piccione and Rubinstein argue that if node $x_{1}$ is reached, the agent should reconsider, and decide to switch from $f$ to $f^{\prime}$. If the agent 
is able to remember that he switched strategies, then this is correct; the agent is indeed better off (under any reasonable notion of "better off") if he switches.

The reason for the time inconsistency here is that an agent's strategy must dictate the same action at nodes $x_{3}$ and $x_{4}$, since they are in the same information set. Intuitively, since the agent cannot distinguish the nodes, he must do the same thing at both. If the agent had perfect recall, he could distinguish the nodes. The optimal strategy with perfect recall amounts to switching from $f$ to $f^{\prime}$ at $x_{1}$ : the agent plays $L$ at $x_{3}$ (as he would with $f^{\prime}$ ) and $R$ at $x_{4}$ (as he would with $f$ ). However, by having the ability to remember that he has switched strategies, the agent is able to simulate perfect recall. If he is using $f^{\prime}$ at the information set, he knows he must have been at $x_{1}$ (and thus is at $\left.x_{3}\right)$; similarly, if he is using $f$ at the information set, then he must be at $x_{4}$. What does it mean, then, to put $x_{3}$ and $x_{4}$ in the same information set? What entitles a modeler to put an ellipse around $x_{3}$ and $x_{4}$ ?

In the computer science literature a different approach is used to represent knowledge in multi-agent systems. This approach goes back to Halpern and Fagin 1989; Halpern and Moses 1990], and has been used quite successfully to model distributed systems applications [Fagin, Halpern, Moses, and Vardi 1995, Chapters 4-7]. The approach can be viewed as combining features of both game trees and the state-space representation. Not surprisingly, it can also be used to model games. The idea is that a game is represented as a multi-agent system. In the description of the system, the actual play of the game is distinguished from what goes on in the agent's mind. I claim that doing so can clear up the type of problems encountered in the game in Figure 3. In the remainder of this section, I describe the approach and sketch how it can be used to deal with this game. I describe some further advantages of the approach in the next section.

\subsection{The multi-agent systems approach}

The basic framework is easy to describe although, unfortunately, the description requires a number of definitions. I review the relevant definitions in this section. 1 To describe the agent's state of mind, we assume that, at every point in time, the agent is in some state. Occasionally this is called a local state, to distinguish it from a global state, which is defined below. The local state is essentially what is called an agent's type in the game theory literature. Intuitively, it encapsulates all the information to which the agent has access. Deciding how to model the state can be quite a nontrivial issue. In a poker game, a player's state might consist of the cards he currently holds, the bets made by the other players, other cards he has seen, and whatever information he has about the strategies of the other players. A forgetful player may not remember all the details of the bets made by the other players; his state would reflect this.

To describe the external world, we use an environment, which is also in some state

${ }^{1}$ The following description is taken almost verbatim from [Halpern 1997. See [Fagin, Halpern, Moses, and Vardi 1995, Chapter 4] for more details. 
at every point in time. Roughly speaking, the environment's state describes everything relevant to the system that is not part of the agents' states. For example, when describing a game, we can take the environment's state at a given point to consist of the sequence of actions that have been performed up to that point; in addition, at points representing the end of play, the environment's state can include the payoffs. If we do this, we can essentially identify the possible environment states with the nodes in the game tree.

The configuration of the system as a whole can be described by a global state, a tuple of the form $\left(\ell_{e}, \ell_{1}, \ldots, \ell_{n}\right)$, where $\ell_{e}$ is the environment's state, and $\ell_{i}$ is agent $i$ 's state, $i=1, \ldots, n$. A global state describes the system at a given point in time. We are typically interested in dynamic systems that change over time. A run is a function from time (which is taken for simplicity to range over the natural numbers) to global states. Intuitively, a run is a complete description of how the system's global state evolves over time. For example, when analyzing a game, a run could be a particular play of the game. Thus, if $r$ is a run, $r(0)$ describes the initial global state of the system, $r(1)$ describes the next global state, and so on. A point is a pair $(r, m)$ consisting of a run $r$ and time $m$. If $r(m)=\left(\ell_{e}, \ell_{1}, \ldots, \ell_{n}\right)$, let $r_{i}(m)=\ell_{i}$. Thus, $r_{i}(m)$ is agent $i$ 's local state at the point $(r, m)$.

Finally, a system is formally defined to be a set of runs. Intuitively, a system is being identified with its set of possible behaviors. Thus, for example, the game of bridge can be identified with all the possible games of bridge that can be played (where a run describes a particular play of the game, by describing the deal of the cards, the bidding, and the play of the hand).

Notice that information sets are conspicuously absent from this definition. Information sets in fact do not have to be specified exogenously; they can be reconstructed from the local states. Given a system, that is, a set $\mathcal{R}$ of runs, we can define an equivalence relation on the points in $\mathcal{R}$. The point $(r, m)$ is indistinguishable from $\left(r^{\prime}, m^{\prime}\right)$ by agent $i$, denoted $(r, m) \sim_{i}\left(r^{\prime}, m^{\prime}\right)$, if $r_{i}(m)=r_{i}^{\prime}(m)$. Thus, two points are indistinguishable by agent $i$ if agent $i$ has the same local state at both points. Clearly $\sim_{i}$ is an equivalence relation. The $\sim_{i}$ relations can be viewed as defining information sets. However, note that even a point where agent $i$ does not move is in an information set for agent $i$. We can now define what it means for agent $i$ to know an event $E$ : agent $i$ knows $E$ at a point $(r, m)$ if the set of points indistinguishable from $(r, m)$ by agent $i$ (that is, $\left.\left\{\left(r^{\prime}, m^{\prime}\right):(r, m) \sim_{i}\left(r^{\prime}, m^{\prime}\right)\right\}\right)$ is a subset of $E$.

A protocol for an agent in this setting is a function from that agent's local states to actions (or to a distribution over actions, if we want to consider randomized protocols). Essentially, a protocol is just a strategy; a randomized protocol is essentially a behavior strategy. The definition captures the intuition that what an agent does can depend only on her information (i.e., her local state). At two points where the agent has the same information, the agent must do the same thing. The agent's local state in this setting corresponds to the agent's information set in the extensive-form representation of the game. However, thinking in terms of local states seems more natural for protocol designers than 
thinking in terms of information states in a game tree. It is much more natural to write a program that says "if you have received a message then send an acknowledgment" than to describe the whole interaction (i.e., the game tree), put an ellipse around those nodes in the game tree in which the agent has received the message (this would be the information set corresponding to the local state where the agent has received the message), and describe the strategy that performs the action of acknowledging the message at that information set. Most importantly, by using local states, it becomes clear exactly what an agent's information is at any point and, thus, what the agent's information set should be.

We often think of systems as generated by agents running a joint protocol, that is, a tuple consisting of a protocol for each agent. Intuitively, starting in some initial global state(s), we run the joint protocol and see what happens step by step. But what exactly happens when a joint protocol $P$ is run? That depends on the setting, or context, in which $P$ is being run. The context determines, among other things, what the environment does. The environment is viewed as running a protocol just like the agents; its protocol is used to capture features of the setting such as "all messages are delivered within 5 rounds" or "messages may be lost". Roughly speaking, the environment's protocol corresponds to "nature's strategy" - the way nature plays the game. The context also determines how the actions performed by the protocol change the global state. Formally, a context $\gamma$ is a tuple $\left(P_{e}, \mathcal{G}_{0}, \tau\right)$, where $P_{e}$ is a protocol for the environment, $\mathcal{G}_{0}$ is a set of initial global states (intuitively, the set of states in which it is possible to start a run), and $\tau$ is a transition function. I $^{2}$ The transition function $\tau$ captures the effect of actions; formally, it describes how the actions performed by the agents and the environment change the global state by associating with each joint action (a tuple consisting of an action for the environment and one for each of the agents) a global state transformer, that is, a mapping from global states to global states.

For ease of exposition, suppose that $P$ is a deterministic joint protocol. A run $r$ is consistent with $P$ in context $\gamma=\left(P_{e}, \mathcal{G}_{0}, \tau\right)$ if the initial global state $r(0)$ is one of the global states in $\mathcal{G}_{0}$, and for all $m$, the transition from global state $r(m)$ to $r(m+1)$ is the result of performing the joint action specified by $P$ and the environment protocol $P_{e}$ in the global state $r(m)$. A system $\mathcal{R}$ represents a joint protocol $P$ in a context $\gamma$ if it consists of all runs in $\Psi$ consistent with $P$ in $\gamma$. (If $P$ is a randomized protocol, essentially the same construction gives the set of runs consistent with $P$ together with a probability distribution on them; I omit the formal details here.)

The role of the context should become clearer in the examples in Section 3 .

\footnotetext{
${ }^{2}$ Often it is also convenient to include in the tuple a component describing which runs are admissible. This is done in Fagin, Halpern, Moses, and Vardi 1995] to capture notions such as fairness: if a message is sent infinitely often, it is eventually received. (Thus, runs where a message is sent infinitely often but not received are considered inadmissible.) Since admissible runs play no role in the discussion here, I omit this component from the context.
} 


\subsection{From game trees to systems}

There is a great deal of flexibility in representing a game using a system. It depends on what the local states are. One possibility essentially directly emulates the extensiveform representation. In this approach, each run in the system correspond to a play of the game - i.e., a branch in the game tree. Thus, there is essentially one run for each terminal node in the game tree. Under this representation, the environment state at a certain point is the node in the game tree (or, equivalently, the sequence of actions taken to reach that node); the environment state at points that correspond to terminal nodes would also include the payoff. An agent's local state could then simply be his information set. A more natural representation of an agent's local state might be the sequence of actions she recalls seeing. ${ }^{3}$

Note that if we represent a game this way, there is no information about strategies, just as there is no information about strategies in the extensive-form representation. This is not due to a lack of expressive power in the systems framework; rather, it is due to the choice of local states.

Another choice is closer to the state-space representation. Each state in a state space corresponds to a run in the system. The play of the game in the run is the play generated by the strategy profile associated with the state. Again, the environment state could be the node reached (and the payoff, if it is a terminal node). But now an agent's local states would include a representation of the strategy she is using, what she recalls having seen thus far, and some representation of her beliefs about other agents' strategies. If it is common knowledge that agents do not switch strategies in the course of the game, this common knowledge can be represented by considering systems that consist only of runs where the players strategy does not change over time.

What happens if agents can switch strategies? Again, there is no difficulty modeling this in the framework. (But note that, strictly speaking, switching strategies should then be considered one of the actions in the game.) An agent's local state would then include her current strategy (or perhaps the sequence of strategies she has used up the current time.) If we model the game in Figure 3 using such a system, if the player knows that he will switch from $f$ to $f^{\prime}$ at $x_{1}$, then at points in the system corresponding $x_{3}$, he will know that he is at $x_{3}$ (because, according to his local state, he is using strategy $f^{\prime}$ ), while at $x_{4}$, he will know that he is at $x_{4}$. If falls right out of the representation that agents that are allowed to switch strategies and know their current strategy will be able to simulate perfect recall.

The key point is that the use of local states in the runs and systems framework forces a modeler to be explicit about what an agent knows and does not know in a way that drawing ellipses in the extensive-form representation or the state-space representation does not. This, in turn, can force some important discipline on the modeler of the

\footnotetext{
${ }^{3}$ Some decision also has to be made as to the agent's local state at nodes where the agent does not move. There are a number of reasonable choices; the one made does not affect the main points.
} 
game. In the game in Figure 3, for example, the modeler is forced to to say whether the player allowed to switch strategies and, if so, whether he keeps track of his current strategy. The answer to this question is modeled in the player's state. Whatever the answer to the question, there will be no time inconsistency. (See Halpern 1997 for a more detailed discussion of this example and the notion of modeling games using the systems framework.)

\section{Coping with Failures and Asynchrony}

There is a great deal of work in the distributed systems literature on designing protocols to deal with certain paradigmatic problems. There is a lot of overlap in spirit between this work and much of the work in the game theory literature on mechanism design. There are, however, also significant differences. Game theory focuses on autonomous agents and their strategic interests. In the distributed systems literature, the "agents" are processes, which are given a protocol to run by a systems designer. The distributed systems literature focuses on what can go wrong and what makes running the protocols difficult - communication failures, process failures, asynchrony, and the complexity issues involved in dealing with large systems. All of these issues are, by and large, not discussed in the game theory literature. In this section, I give examples of problems in which issues of failures and asynchrony arise. These examples also illustrate some other advantages of using the systems representation.

\subsection{Coordinated Attack}

The coordinated attack problem is a well-known problem from the distributed systems folklore [Gray 1978]. The following description of the problem is taken from [Halpern and Moses 1990; the discussion of it is taken from [Halpern 1995].

Two divisions of an army are camped on two hilltops overlooking a common valley. In the valley awaits the enemy. It is clear that if both divisions attack the enemy simultaneously they will win the battle, whereas if only one division attacks it will be defeated. The generals do not initially have plans for launching an attack on the enemy, and the commanding general of the first division wishes to coordinate a simultaneous attack (at some time the next day). Neither general will decide to attack unless he is sure that the other will attack with him. The generals can only communicate by means of a messenger. Normally, it takes the messenger one hour to get from one encampment to the other. However, it is possible that he will get lost in the dark or, worse yet, be captured by the enemy. Fortunately, on this particular night, everything goes smoothly. How long will it take them to coordinate an attack? 
In the language of game theory, the problem here is to design a mechanism that guarantees that the generals coordinate, despite the possibility of messages being lost. As is typically the case in distributed systems problems, there is no discussion of what the payoff is for general $A$ and $B$ if both attack, neither does, or one does and the other does not. Nor is there is any mention of probabilities (in particular, the probability that the messenger will arrive). While interesting issues certainly arise if strategic concerns and probability are added (see, for example, Rubinstein's [1989] lovely results), there are good reasons why these issues are being ignored here. The coordinated attack problem is an attempt to understand the effect of possible communication failures on coordination. The generals are viewed as being on the same "team", with identical utilities, playing against "nature" or the "environment", which controls communication. We could capture the intuition behind the problem by giving each general payoff $L$ if they do not coordinate, utility $M$ if neither attacks, and utility $H$ if both attack, with $L<M<H$, but no new issues would arise if we did so. The real interest here is not in the strategic behavior of the generals, but whether they can achieve coordination when playing against nature.

We could also add a probability that a message arrives. The problem is that, for the situations which the coordinated attack problem was intended to abstract, it is often quite difficult to characterize this probability. For example, one reason that messages fail to arrive in real systems is message congestion, often caused by "hotspots". The probability of message congestion is extremely difficult to characterize.

Turning to the analysis of the problem, suppose that the messenger sent by General $A$ makes it to General $B$ with a message saying "Let's attack at dawn." Will General $B$ attack? Of course not, since $A$ does not know that $B$ got the message, and thus may not attack. So $B$ sends the messenger back with an acknowledgment. Suppose the messenger makes it. Will $A$ attack? No, because now $B$ does not know that $A$ got the message, so $B$ thinks $A$ may think that he $(B)$ didn't get the original message, and thus not attack. So $A$ sends the messenger back with an acknowledgment. But of course, this is not enough either.

In terms of knowledge, each time the messenger makes a transit, the depth of the generals' knowledge increases by one. More precisely, let $E$ be the event "a message saying 'Attack at dawn' was sent by General $A$ ". When General $B$ gets the message, $B$ knows $E$. When $A$ gets $B$ 's acknowledgment, $A$ knows that $B$ knows $E$. Every pair of subsequent acknowledgment leads to one more level of " $A$ knows that $B$ knows." However, although more acknowledgments keep increasing the depth of knowledge, it is not hard to show that by following this protocol, the generals never attain common knowledge that the attack is to be held at dawn, where common knowledge describes the event that $A$ knows that $B$ knows that $A$ knows that $B$ knows ad infinitum.

What happens if the generals use a different protocol? That does not help either. As long as there is a possibility that the messenger may get captured or lost, then common knowledge is not attained, even if the messenger in fact does deliver his messages. It would take us too far afield here to completely formalize these results (see Fagin, Halpern, 
Moses, and Vardi 1995, Section 6.1] for details), but it is not hard to give a rough description. A context $\gamma$ displays unbounded message delays (umd) if, roughly speaking, for all systems $\mathcal{R}$ that represent a protocol $P$ run in context $\gamma$, runs $r \in \mathcal{R}$, and agents $i$, if $i$ receives a message at time $m$ in $r$, then for all $m^{\prime}>m$, there is another run $r^{\prime} \in \mathcal{R}$ that is identical to $r$ up to time $m$ except that agent $i$ receives no messages in $r^{\prime}$ between times $m$ and $m^{\prime}$ inclusive, and no agent other than possible $i$ can distinguish $r$ and $r^{\prime}$ up to time $m^{\prime}$ (i.e., $r_{j}\left(m^{\prime \prime}\right)=r_{j}^{\prime}\left(m^{\prime \prime}\right)$ for $m^{\prime \prime} \leq m^{\prime}$ and $j \neq i$ ). That is, $r^{\prime}$ looks the same as $r$ up to time $m^{\prime}$ to each agent except possibly $i$, and all messages that $i$ receives in $r$ between times $m$ and $m^{\prime}$ are delayed until after time $m^{\prime}$ in $r^{\prime}$. We can think of umd as characterizing a property of the environment's protocol in context $\gamma$. Intuitively, it is the environment that decides whether or not a message is delivered; in a context with umd, the environment is able to hold up messages for an arbitrary amount of time.

Theorem 3.1: Halpern and Moses 1990 If context $\gamma$ displays umd and $\mathcal{R}$ is a system that represents some protocol $P$ in context $\gamma$, then at no point in $\mathcal{R}$ can it be common knowledge that a message has been delivered.

This says that, in a context that displays umd, no matter how many messages arrive, the generals cannot attain common knowledge that any message whatsoever has been delivered. Since it can never become common knowledge that a message has been delivered, and message delivery is a prerequisite for attack, it is not hard to show that it can never become common knowledge among the generals that they are attacking. More precisely, let attack be the event that consists of the points where both generals attack.

Corollary 3.2: If context $\gamma$ displays umd and $\mathcal{R}$ is a system that represents some protocol $P$ in context $\gamma$, then at no point in $\mathcal{R}$ can attack be common knowledge among the generals.

Why is it relevant that the generals can never get common knowledge of the fact that they are attacking? Our interest here is not common knowledge, but coordinated attack. What does common knowledge have to do with coordinated attack? As the next result shows, a great deal. Common knowledge is a prerequisite for coordination. Let a system for coordinated attack be one that represents a protocol for coordinated attack.

Theorem 3.3: Halpern and Moses 1990] In a system for coordinated attack, when the generals attack, attack must be common knowledge among the generals.

The statement of the coordinated attack problem assumes that the generals have no initial plans for attack. This can be formalized by assuming that, in the absence of messages, they will not attack. With this assumption, Corollary 3.2 and Theorem 3.3 together give the following result. 
Corollary 3.4: If context $\gamma$ displays umd and $\mathcal{R}$ is a system that represents some protocol for coordinated attack in context $\gamma$, then at no point in $\mathcal{R}$ do the generals attack.

Note that this result can be expressed in game theoretic terms: it is impossible to design a mechanism that guarantees coordinated attack. These results show not only that coordinated attack is impossible (a fact that was well known [Yemini and Cohen 1979]), but why it is impossible. The problem is due to a combination of (1) the unattainability of common knowledge in certain contexts and (2) the need for common knowledge to perform coordination.

It is worth stressing the role of systems and contexts in stating these results. The notion of "communication not being guaranteed" was formulated in terms of a condition on contexts (umd). Theorem 3.1 show that common knowledge in any system that can be generated in a context satisfying umd. The need for common knowledge to coordinate is also formulated in terms of systems. The framework of runs and systems is well suited to formulating these conditions.

\subsection{Byzantine Agreement}

The coordinated attack problem focused on communication problems. Byzantine agreement is another paradigmatic problem in the distributed systems literature; it brings out issues of process failures as well as asynchrony. In this problem, there are assumed to be $n$ soldiers, up to $t$ of which may be faulty (the $t$ stands for traitor); $n$ and $t$ are assumed to be common knowledge. Each soldier starts with an initial preference, to either attack or retreat. (More precisely, there are two types of nonfaulty agents - those that prefer to attack, and those that prefer to retreat.) We want a protocol (i.e., a mechanism) with the following properties:

- All nonfaulty soldiers reach the same decision.

- If all the soldiers are nonfaulty and their initial preferences are identical, then the final decision agrees with their initial preferences.t

This problem has been studied in detail. There have been literally hundreds of papers on Byzantine agreement and closely related topics. The problem was introduced by Pease, Shostak, and Lamport [1980]; Fischer [1983] gives an overview of the state of the art in the early 1980's; Linial [1994] gives a more recent discussion; Chor and Dwork [1989 survey randomized algorithms for Byzantine agreement. Whether the Byzantine agreement problem is solvable depends in part on what types of failures are considered, on whether the system is synchronous or asynchronous, and on the ratio of $n$ to $t$. Roughly

\footnotetext{
${ }^{4}$ This condition simply prevents the obvious trivial solutions, where the soldiers attack no matter what, or retreat no matter what. Similarly, the statement "The generals do not initially have plans to attack" in the description of the coordinated attack problem is implicitly meant to prevent a similar trivial solution in the case of coordinated attack.
} 
speaking, a system is synchronous if there is a global clock and agents move in lockstep; a "step" in the system corresponds to a tick of the clock. In an asynchronous system, there is no global clock. The agents in the system can run at arbitrary rates relative to each other. One step for agent 1 can correspond to an arbitrary number of steps for agent 2 and vice versa. Synchrony is an implicit assumption in essentially all games. Although it is certainly possible to model games where player 2 has no idea how many moves player 1 has taken when player 2 is called upon to move, it is certainly not typical to focus on the effects of synchrony (and its lack) in games. On the other hand, in distributed systems, it is typically a major focus.

Byzantine agreement is achievable in certain cases. Suppose that the only types of failures are crash failures - a faulty agent behaves according to the protocol, except that it might crash at some point, after which it sends no messages. In the round in which an agent fails, the agent sends only a subset of the messages that it is supposed to send according to its protocol. Further suppose that the system is synchronous. (These two assumptions can be captured by considering the appropriate context; see Fagin, Halpern, Moses, and Vardi 1995, p. 203].) In this case, the following rather simple protocol achieves Byzantine agreement:

- In the first round, each agent tells every other agent its initial preference.

- For rounds 2 to $t+1$, each agent tells every other agent everything it has heard in the previous round. (Thus, for example, in round 3, agent 1 may tell agent 2 that it heard from agent 3 that its initial preference was to attack, and that it (agent 3) heard from agent 2 that its initial preference is to attack, and it heard from agent 4 that its initial preferences is to retreat, and so on. This means that messages get exponentially long, but it is not difficult to represent this information in a compact way so that the total communication is polynomial in $n$, the number of agents.)

- At the end of round $t+1$, if an agent has heard from any other agent (including itself) that its initial preference was to attack, it decides to attack; otherwise, it decides to retreat.

Why is this correct? Clearly, if all agents are correct and want to retreat, then the final decision will be to retreat, since that is the only preference that other agents hear about (recall that for now we are considering only crash failures). Similarly, if all agents prefer to attack, the final decision will clearly be to attack. It remains to show that if some agents prefer to attack and others to retreat, then all the nonfaulty agents reach the same final decision. So suppose that $i$ and $j$ are nonfaulty and $i$ decides to attack. That means that $i$ heard that some agent's initial preference was to attack. If it heard this first at some round $t^{\prime}<t+1$, then $i$ will forward this message to $j$, who will receive it and thus also attack. On the other hand, suppose that $i$ heard it first at round $t+1$ in a message from $i_{t+1}$. Thus, this message must be of the form " $i_{t}$ said at round $t$ that ... that $i_{2}$ said at round 2 that $i_{1}$ said at round 1 that its initial preference was 
to attack." Moreover, the agents $i_{1}, \ldots, i_{t+1}$ must all be distinct. Indeed, it is easy to see that $i_{k}$ must crash in round $k$ before sending its message to $i$ (but after sending its message to $i_{k+1}$ ), for $k=1, \ldots, t$, for otherwise $i$ must have gotten the message from $i_{k}$, contradicting the assumption that $i$ first heard at round $t+1$ that some agent's initial preference was to attack. Since at most $t$ agents can crash, it follows that $i_{t+1}$, the agent that sent the message to $i$, is not faulty, and thus sends the message to $j$. Thus, $j$ also decides to attack. A symmetric argument shows that if $j$ decides to attack, then so does $i$.

It should be clear that the correctness of this protocol depends on both the assumptions made: crash failures and synchrony. Suppose instead that Byzantine failures are allowed, so that faulty agents can deviate in arbitrary ways from the protocol; they may "lie", send deceiving messages, and collude to fool the nonfaulty agents in the most malicious ways. In this case, the protocol will not work at all. In fact, it is known that agreement can be reached in the presence of Byzantine failures iff $t<n / 3$, that is, iff fewer than a third of the agents can be faulty Pease, Shostak, and Lamport 1980]. The effect of asynchrony is even more devastating: in an asynchronous system, it is impossible to reach agreement using a deterministic protocol even if $t=1$ (so that there is at most one failure) and only crash failures are allowed [Fischer, Lynch, and Paterson 1985]. The problem in the asynchronous setting is that if none of the agents have heard from, say, agent 1, they have no way of knowing whether agent 1 is faulty or just slow. Interestingly, there are randomized algorithms (i.e., behavior strategies) that achieve agreement with arbitrarily high probability in an asynchronous setting [Ben-Or 1983; Rabin 1983.

Finally, note that the protocol above uses $t+1$ rounds. This bound is achievable even with Byzantine failures, provided that that $t<n / 3$ Pease, Shostak, and Lamport 1980. Can we do better? In one sense, the answer is no. Even if only crash failures are considered, $t+1$ rounds of communication are required in runs where there are in fact no failures at all [Dolev and Strong 1982]. To understand why, consider a simple situation where $t=1$, there are only crash failures, all agents start with the same initial preference, say to attack, and there are in fact no failures. In this case, all the agents can tell each other in the first round of communication that they want to attack. Since there are no failures, at the end of the first round, all the agents will know that all the other agents want to attack. Thus, they will know that the ultimate decision must be to attack, since all the agents have the same initial preference. Nevertheless, if they want to be sure to attack simultaneously, they must wait until the end of the second round to do so ( since $t+1=2$ in this case).

Why is this the case? Results of Dwork and Moses [1990 give some insight here. They show that common knowledge among the nonfaulty agents is necessary and sufficient to attain simultaneous Byzantine agreement (even though a nonfaulty agent may not know which of the other agents are faulty). The nonfaulty agents are what is called an indexical set in the philosophy literature; a set whose membership depends on context. The reason it takes two rounds to reach agreement even if there are no failures is that, although each agent knows that all the other agents had an initial preference to attack, this fact is not 
yet common knowledge. For example, agent 1 might consider it possible that agent 2 was faulty and crashed before sending a message to agent 3 . In this case, agent 3 would not know that everyone started with an initial preference to attack. Moreover, in this case, agent 3 might consider it possible that agent 2's initial preference was to retreat, and that agent 2 communicated this preference to agent 1 . This argument can be extended to show that agent 1 considers it possible that agent 3 considers it possible that agent 1 considers it possible ... that everyone's initial preference was to retreat.

It might seem that if it takes $t+1$ rounds to reach simultaneous agreement in the case that there are no failures, then things can only get worse if there are failures. However, Dwork and Moses show that this intuition is misleading. They use their characterization of agreement to provide algorithms for simultaneous Byzantine agreement that reach agreement as early as possible, as a function of the pattern of failures. Roughly speaking, we can imagine an adversary with $t$ "chips", one for each possible failure. The adversary plays a chip by corrupting an agent. Dwork and Moses' analysis shows that if the adversary's goal is to make the agreement happen as late as possible, then the adversary's optimal strategy is, roughly speaking, to play no more than one chip per round. If the adversary plays optimally, agreement cannot be attained before round $t+1$. Since not corrupting any agent is an instance of optimal play, it follows that it requires $t+1$ rounds to reach agreement in runs where there are no failures. On the other hand, if the adversary plays all $t$ chips in the first round and none of the faulty agents sends a message, then the correct agents will know at the end of the first round exactly which agents are faulty, and be able to reach agreement in one more round. The adversary is best off by keeping the agents as uncertain as possible as to which agents are faulty.

Byzantine agreement can be viewed as a game where, at each step, an agent can either send a message or decide to attack or retreat. It is essentially a game between two teams, the nonfaulty agents and the faulty agents, whose composition is unknown (at least by the correct agents). To model it as a game in the more traditional sense, we could imagine that the nonfaulty agents are playing against a new player, the "adversary". One of adversary's moves is that of "corrupting" an agent: changing its type from "nonfaulty" to "faulty". Once an agent is corrupted, what the adversary can do depends on the failure type being considered. In the case of crash failures, the adversary can decide which of a corrupted agent's messages will be delivered in the round in which the agent is corrupted; however, it cannot modify the messages themselves. In the case of Byzantine failures, the adversary essentially gets to make the moves for agents that have been corrupted; in particular, it can send arbitrary messages.

In practice, crash failures occur quite regularly, as a result of hardware and software failures. Another failure type considered is omission failures. An agent suffering from an omission failure behaves according to its protocol, except that it may omit to send an arbitrary set of messages in any given round. Omission failures are meant to model local communications problems (for example, a congested message buffer). Finally, Byzantine failures represent the worst possible failures, where we can make no assumption on the behavior of faulty agents. Byzantine failures are used to capture random behavior on the 
part of a system (for example, messages getting garbled in transit), software errors, and malicious adversaries (for example, hackers).

In the case of crash failures and omission failures (and for Byzantine failures that are meant to represent random behavior), it does not make sense to view the adversary's behavior as strategic, since in these cases the adversary is not really viewed as having strategic interests. However, it would certainly make sense, at least in principle, to consider the probability of failure (i.e., the probability that the adversary corrupts an agent). But this approach has by and large been avoided in the literature. It is very difficult to characterize the probability distribution of failures over time. Computer components can perhaps be characterized as failing according to an exponential distribution (as is done by Babaoglu [1987], in one of the few papers that I am aware of that actually does try to analyze the situation probabilistically), but crash failures can be caused by things other than component failures (faulty software, for example). Omission failures are often caused by traffic congestion; as I mentioned before, this is extremely difficult to characterize probabilistically. The problems are even worse when it comes to modeling random Byzantine behavior.

With malicious Byzantine behavior, it may well be reasonable to impute strategic behavior to agents (or to an adversary controlling them). However, it is typically very difficult to characterize the payoffs of a malicious agent (and, indeed, there is often a great deal of uncertainty about what a malicious agent's payoffs are). The goals of the agents may vary from that of simply trying to delay a decision to that of causing disagreement. It is not clear what the appropriate payoffs should be for attaining these goals. Thus, the distributed systems literature has chosen to focus instead on algorithms that are guaranteed to satisfy the specification without making assumptions about the adversary's payoffs (or nature's probabilities, in the case of omission failures and crash failures).

I believe that some interesting work can be done trying to combine failures, asynchrony, and strategic incentives. Some preliminary work has already been done - for example, Monderer and Tennenholtz [1999a, 1999b have considered timing issues in asynchronous systems, as well as the structure of the network, and Eliaz 2000 has considered solution concepts that take failures into account. However, I believe that there is much more that can be done.

\section{Specification and Mechanism Design}

Game theory has typically focused on "small" games: games that are easy to describe, such as Prisoner's Dilemma, Battle of the Sexes, and the Centipede game. The focus has been on subtleties regarding basic issues such as rationality and coordination. To the extent that game theory is used to tackle larger, more practical problems, and especially to the extent that it is computers, or software agents, playing games, rather than people, a whole host of new issues arise. In many cases, the major difficulty may no longer be 
conceptual problem of explicating what ought to be considered "rational". It may be quite obvious what the "rational" and optimal strategy is once we analyze the game. Rather, the difficulty is analyzing the game due to its size. Indeed, part of the difficulty might even be describing the game. By way of analogy, $2^{n}-1$ numbers are needed to describe a probability distribution on a space characterized by $n$ binary random variables. For $n=100$ (not an unreasonable number in practical situations), it is impossible to write down the probability distribution in the obvious way, let alone do computations with it. The same issues will surely arise in large games. Computer scientists have developed techniques like Bayesian networks for manipulating probability measures on large spaces Pearl 1988; similar techniques seem applicable to games. Since these techniques are discussed in detail by Koller and Milch [2001] and La Mura [2000], I do not go into them here.

A related but different problem is involved with dealing with "large" mechanisms. This, I expect, will be somewhat akin to writing large programs. It will be extremely important to specify carefully exactly what the mechanism must accomplish, and to find techniques for doing mechanism design in a modular way, so that mechanisms for solving different problems can be combined in a seamless way.

The design and specification of software is well known to be a critical and often difficult problem in computer science. The concern with specification has led to the development of numerous specification languages, that is, formal languages for expressing carefully the requirements that a protocol must satisfy (see, for example, Harel, Kozen, and Tiuryn 2000; Milner 1980; Manna and Pnueli 1992]).

It may seem that specification is not so hard. How hard is it, for example, to specify a division algorithm? It gets two inputs $x$ and $y$ and is supposed to return $x / y$. Even in this simple case, there are difficulties. Are we talking about integer division (so that the inputs are integers and the output is an integer, with the remainder ignored)? If we are talking about real division, how should the the answer be represented? For example, if it is a decimal, what should the answer be if $x$ and $y$ are 1 and 3, respectively? How is the infinite sequence $.333 \ldots$ to be represented? If answers can only be, say, 32 bits long, what happens if $x=10^{31}$ and $y=10^{-31}$ ? What should happen if $y=0$ ? And what happens if the inputs are not of the right type (i.e., they are letters instead of numbers)? The key point is that a good specification will need to describe what should happen in all the "unexpected" cases.

This can get particularly difficult once we try to take into account failures and asynchrony. Imagine trying to specify a good mechanism for a distributed auction. The specification will need to take into account the standard distributed concerns of asynchronous communication, failures, garbled communication, economic issues like failure to pay, and strategic issues (including strategic uses of computing difficulties, such as pretending not to have received messages or to have received them late). Thus, its speci-

\footnotetext{
${ }^{5}$ Historically, the original work that my colleagues and I did on knowledge and common knowledge was in part motivated by the desire to find good tools for designing and specifying distributed protocols.
} 
fication must address what should happen if a process fails in the middle of transmitting a bid, how to deal with agents bidding on slow lines, and so on.

Things get significantly more complicated if we try to specify notions like security. What exactly does it mean that a mechanism is secure? What types of attacks can be tolerated? For example, how should the mechanism behave if there is a denial-of-service attack? I suspect that questions regarding security and fault-tolerance will turn out to be closely intertwined with strategic issues. Thus, finding appropriate techniques for specifying mechanisms will not simply be a matter of lifting standard techniques from software specification.

\section{Conclusions}

I have focused on one set of issues at the interface of computer science and game theory here, which arise from work in distributed computing. As I hope this discussion has made clear, I think that game theorists need to take more seriously issues like fault tolerance, asynchrony, the representation of knowledge and uncertainty, the difficult in the design and analysis of large mechanisms and games, and problems of specification. On the other hand, I think computer scientists need to take strategic concerns more seriously in the design and analysis of distributed protocols. These issues are not just of theoretical interest. They arise, for example, when we consider the design of Internet agents. We will certainly need to take into account failures, and no company would want to claim to support software for agents that bid in auctions that has not been carefully specified.f The specification of the agents will, in turn, depend on a careful specification of the mechanism in which they will be participating.

These issues represent only part of the commonality in interests that I see between computer science and game theory. I have already hinted at another important area of commonality: that of finding compact representations of games. Other issues of common interest include learning, mental-level modeling of beliefs Brafman and Tennenholtz 1997, qualitative decision theory (see the bibliography of over 290 papers at http://www.medg.lcs.mit.edu/qdt/bib/unsorted.bib). With the growing awareness of the commonality between computer science and game theory, I look forward to a great deal of fruitful interaction between the fields in the coming years.

\section{Acknowledgments}

I would like to thank Ron Fagin, Yoram Moses, Ariel Rubinstein, Moshe Tennenholtz, and Moshe Vardi for useful comments on an earlier draft of this paper.

\footnotetext{
${ }^{6}$ The correctness of the agents will also have to be verified somehow. Verification is yet another issue of great concern in computer science that may prove relevant to game theory. Much work has gone into finding (preferably automatic or semi-automatic) techniques to check that a protocol satisfies a specification (see, for example, Apt and Olderog 1991; Clarke, Grumberg, and Peled 1999).
} 


\section{References}

Apt, K. R. and E.-R. Olderog (1991). Verification of sequential and concurrent programs. New York: Springer-Verlag.

Aumann, R. J. (1976). Agreeing to disagree. Annals of Statistics 4 (6), 1236-1239.

Babaoglu, O. (1987). On the reliability of consensus-based fault-tolerant distributed computing systems. ACM Transactions on Computer Systems 5, 394-416.

Ben-Or, M. (1983). Another advantage of free choice: completely asynchronous agreement protocols. In Proc. 2nd ACM Symp. on Principles of Distributed Computing, pp. 27-30.

Brafman, R. I. and M. Tennenholtz (1997). Modeling agents as qualitative decisionmakers. Artificial Intelligence 94, 217-268.

Chor, B. and C. Dwork (1989). Randomization in Byzantine agreement. In Advances in Computing Research 5: Randomness and Computation, pp. 443-497. JAI Press.

Clarke, E. M., O. Grumberg, and D. A. Peled (1999). Model Checking. Cambridge, Mass.: MIT Press.

Dolev, D. and H. R. Strong (1982). Requirements for agreement in a distributed system. In H. J. Schneider (Ed.), Distributed Data Bases, pp. 115-129. Amsterdam: NorthHolland.

Dwork, C. and Y. Moses (1990). Knowledge and common knowledge in a Byzantine environment: crash failures. Information and Computation 88(2), 156-186.

Eliaz, K. (2000). Fault-tolerant implementation. Unpublished manuscript.

Fagin, R., J. Y. Halpern, Y. Moses, and M. Y. Vardi (1995). Reasoning about Knowledge. Cambridge, Mass.: MIT Press.

Fischer, M. J. (1983). The consensus problem in unreliable distributed systems. Technical Report RR-273, Yale University. Also appears in Foundations of Computation Theory, ed. M. Karpinski, Lecture Notes in Computer Science, Vol. 185, Springer Verlag, 1983, pp. 127-140.

Fischer, M. J., N. A. Lynch, and M. S. Paterson (1985). Impossibility of distributed consensus with one faulty processor. Journal of the ACM 32(2), 374-382.

Gray, J. (1978). Notes on database operating systems. In R. Bayer, R. M. Graham, and G. Seegmuller (Eds.), Operating Systems: An Advanced Course, Lecture Notes in Computer Science, Vol. 66. Berlin/New York: Springer-Verlag. Also appears as IBM Research Report RJ 2188, 1978.

Halpern, J. Y. (1995). Reasoning about knowledge: a survey. In D. M. Gabbay, C. J. Hogger, and J. A. Robinson (Eds.), Temporal and Epistemic Reasoning, Volume 4 of Handbook of of Logic in Artificial Intelligence and Logic Programming, pp. 1-34. Oxford, U.K.: Oxford University Press. 
Halpern, J. Y. (1997). On ambiguities in the interpretation of game trees. Games and Economic Behavior 20, 66-96.

Halpern, J. Y. and R. Fagin (1989). Modelling knowledge and action in distributed systems. Distributed Computing 3(4), 159-179. A preliminary version appeared in Proc. 4th ACM Symposium on Principles of Distributed Computing, 1985, with the title "A formal model of knowledge, action, and communication in distributed systems: preliminary report".

Halpern, J. Y. and Y. Moses (1990). Knowledge and common knowledge in a distributed environment. Journal of the ACM 37(3), 549-587. A preliminary version appeared in Proc. 3rd ACM Symposium on Principles of Distributed Computing, 1984.

Harel, D., D. C. Kozen, and J. Tiuryn (2000). Dynamic Logic (Foundations of Computing). Cambridge, Mass.: MIT Press.

Hintikka, J. (1962). Knowledge and Belief. Ithaca, N.Y.: Cornell University Press.

Kfir-Dahav, N. E., D. Monderer, and M. Tennenholtz (2000). Mechanism design for resource bounded agents. Unpublished manuscript.

Koller, D. and B. Milch (2001). Structured models for multiagent interactions. In Theoretical Aspects of Rationality and Knowledge: Proc. Eighth Conference (TARK2001), pp. 233-248. San Francisco, Calif.: Morgan Kaufmann.

La Mura, P. (2000). Game networks. In Proc. Sixteenth Conference on Uncertainty in Artificial Intelligence (UAI 2000).

Linial, N. (1994). Games computers play: game-theoretic aspects of computing. In R. J. Aumann and S. Hart (Eds.), Handbook of Game Theory with Economic Applications, Volume II, pp. 1340-1395. Amsterdam: North-Holland.

Manna, Z. and A. Pnueli (1992). The Temporal Logic of Reactive and Concurrent Systems, Volume 1. Berlin/New York: Springer-Verlag.

Milner, R. (1980). A Calculus of Communicating Systems. Lecture Notes in Computer Science, Vol. 92. Berlin/New York: Springer-Verlag.

Monderer, D. and M. Tennenholtz (1999a). Distributed games. Games and Economic Behavior 28, 55-72.

Monderer, D. and M. Tennenholtz (1999b). Distributed Games: From Mechanisms to Protocols. In AAAI-99, pp. 32-37.

Neyman, A. (1985). Bounded complexity justifies cooperation in finitely repated prisoner's dilemma. Economic Letters 19, 227-229.

Pearl, J. (1988). Probabilistic Reasoning in Intelligent Systems. San Francisco, Calif.: Morgan Kaufmann.

Pease, M., R. Shostak, and L. Lamport (1980). Reaching agreement in the presence of faults. Journal of the ACM $27(2), 228-234$. 
Piccione, M. and A. Rubinstein (1997). On the interpretation of decision problems with imperfect recall. Games and Economic Behavior 20(1), 3-24.

Rabin, M. O. (1983). Randomized Byzantine generals. In Proc. 24th IEEE Symp. on Foundations of Computer Science, pp. 403-409.

Rubinstein, A. (1986). Finite automata play the repeated prisoner's dilemma. Journal of Economic Theory 39, 83-96.

Rubinstein, A. (1989). The electronic mail game: strategic behavior under "almost common knowledge". American Economic Review 79, 385-391.

Yemini, Y. and D. Cohen (1979). Some issues in distributed processes communication. In Proc. of the 1st International Conf. on Distributed Computing Systems, pp. 199-203. 